\title{
Crystallographic texture evolution in 1008 steel sheet during multi-axial tensile strain paths
}

\author{
Adam Creuziger ${ }^{1 *}$, Lin Hü ${ }^{2,3}$, Thomas Gnäupel-Herold ${ }^{1}$ and Anthony D Rollett ${ }^{2}$
}

\author{
${ }^{*}$ Correspondence: \\ adam.creuziger@nist.gov \\ ${ }^{1}$ National Institute of Standards and \\ Technology, 100 Bureau Dr., 20899, \\ Gaithersburg, MD, USA \\ Full list of author information is \\ available at the end of the article
}

\begin{abstract}
This paper considers the crystallographic texture evolution in a 1008 low carbon steel. The texture evolution along uniaxial, plane strain and balanced biaxial strain states were measured. For uniaxial testing, grains tend to rotate such that the $\{111\}\langle 1 \overline{1} 0\rangle$ slip directions are aligned with the loading axis. For plane strain and balanced biaxial strain states, the majority of grains are distributed with the $\{111\}$ plane parallel to the sample normal direction. Accompanying visco-plastic self consistent (VPSC) predictions of the texture evolution were made along same strain paths and strain increments.

Comparing between the measured texture evolution and computational texture evolution indicate that the VPSC model qualitatively predicts the measured texture evolution, but the rate at which the texture evolution occurs is over predicted.
\end{abstract}

Keywords: Crystallographic texture; Steel; Neutron diffraction; Metal forming

\section{Introduction}

Steel has been in use for nearly 4000 years, and remains one of the main materials (along with concrete, glass and asphalt) used around the world. A large majority of the steels created are carbon steels, with a large proportion of these carbon steels strained after production to create a required shape. Accurate prediction of the final shape and mechanical properties after straining continues to be an important industrial goal. One of the methods applied to this problem is measurement of the crystallographic texture (or preferred orientation) as a function of strain path and strain increment. Accurate prediction of the crystallographic texture and how the texture evolves will allow better estimates of the flow stress and plastic contraction, which in turn provides a more accurate prediction of the final shape and failure during forming. The particular case of deformation of sheet stock material is discussed in this paper. The majority of the literature discusses the high strain increments relevant for rolling sheet material. Unfortunately existing [1-6] experimental data on the texture evolution along multiaxial strain states at intermediate strain increments $(\Delta \epsilon)$ relevant for forming are incomplete, so assessment of model accuracy is difficult in this regime.

This paper focuses on the measurement of the crystallographic texture evolution in $1008 \mathrm{AISI} / \mathrm{SAE}$ low carbon steel sheets after strain increments typical in forming structures from the rolled sheet. The texture is measured by neutron diffraction to interrogate a large number of grains. Five different strain states were investigated: uniaxial strain along 
the rolling direction (URD), strain along the rolling direction with the transverse direction fixed near zero strain (a plane strain condition, or PSRD), balanced biaxial strain (BB) in the rolling and transverse directions, strain along the transverse direction with the rolling direction fixed near zero strain (PSTD), and uniaxial strain along the transverse direction (UTD). In the plane of the sheet (normal direction or ND) the sample is assumed to be in a plane stress condition. In addition, the texture evolution is predicted from the as received texture using the visco-plastic self consistent (VPSC) model and compared with the experimental data. Data on the texture evolution of low carbon steels will also provide a well characterized reference for comparison to more complicated materials, such as high strength steels (HSS) like high strength low alloy (HSLA) steel or advanced high strength steels (AHSS) such as dual phase (DP) or transformation induced plasticity (TRIP) steels.

The data described in this paper has been submitted to the NIST/MatDL DSpace repository as part of the IMMI General Articles Collection [7]. The repository contains files that include a description of the data contained in the repository, a schematic of the the analysis and computational processes, some of the programs used to analyze the data, a collection of the texture data files at various points in the analysis, and input files to the VPSC model.

\section{Background}

Using the American Iron and Steel Institute (AISI) and Society for Automotive Engineers (SAE) grade designation, 1008 steel is defined as an iron alloy possessing a mass fraction of carbon $\leq 0.0010$, a mass fraction of manganese between 0.0030 and 0.0050 , a mass fraction of phosphorus $\leq 0.0004$, and a mass fraction of sulfur $\leq 0.0005$ [8]. Owing to the low carbon content and absence of austenitic stabilizers, the structure is almost entirely ferritic (body centered cubic crystal structure) with some carbides. This allows for high ductility (approximately 40\% elongation), but low strength (tensile strength approximately $350 \mathrm{MPa}$ ) [9].

The texture evolution of steels has been widely studied in the past, particularly with respect to the complete processing path from the melt to hot and cold rolling. These topics are of interest to create desirable textures for deep drawing. Some articles on these topics include $[2,3,10,11]$, which discuss steel textures and their origin from deformation and recrystalization of the austenite phase and subsequent transformation from austenite to ferrite. The complex details of the austenitic transformation are beyond the scope of this paper, but it is important to note that deformation and recrystallization in the austenite phase are one of the primary causes of crystallographic texture in the ferritic phase. The review by Inagaki [12] summarizes work on the inter-relationship between texture, grain boundaries and dislocation structures in low carbon steels. Hutchinson [13] discusses texture control in low carbon steels as a function of processing parameters. Bodin et al. [2] shows the strong effects of processing path and temperature on the texture.

The predicted plastic strain ratio ( $R$ value) has been used to correlate the effect of a particular texture component to the macroscopic properties. For deep drawing applications, sheet material that has a large volume of grains aligned with the $\gamma$-fiber ( $\{111\}$ plane parallel to the ND) are preferred as they have a high average $\mathrm{R}$ value $(\bar{R})$ and a planar anisotropy $(\Delta R)$ value near $0[10,13]$. Orientations with the $\{100\}$ plane parallel to the ND direction such as 'cube' and 'shear' orientations are not desirable, since they have low average R values $[10,13]$. This is due to the orientation of slip systems for body centered cubic (BCC) 
materials. Conversely, for high strength materials where high ductility is not required, orientations along the $\alpha$-fiber ( $\langle 110\rangle$ crystal direction parallel to rolling direction) are preferred for high strength [14] but may produce anisotropy and brittleness, particularly for the $\{113\}\langle 110\rangle$ orientation [10].

There is also a significant amount of investigation into the textures caused by cold rolling in the ferrite phase. This data is particularly relevant to this paper as cold rolling, apart from a hydrostatic term, produces a stress state similar to plane strain along the rolling direction (PSRD). As shown in a series of review papers, cold rolling ferrite strengthens the $\alpha$ and $\gamma$ fibers, particularly the $\alpha$ fiber at higher reduction ratios [3,10,11]. Figure thirteen in Kestens and Jonas [11] shows relative amounts of texture intensity as a function of thickness reduction. Inagaki [12] discusses some of the microstructural reasons for this, and theorizes that grain pairs with $\{111\}[\mathrm{uvw}]$ type grain boundaries that allow the grain to rotate easily, keeping orientations along the $\gamma$ fiber stable.

Kestens and Jonas [11] proposes that $\{211\}\langle 011\rangle$ along the $\alpha$ fiber is the stable final orientation in plane strain compression, while $\{111\}\langle 110\rangle$ and $\{554\}\langle 225\rangle$ orientations are caused by ferritic recrystallization after plane strain compression. Inagaki [12] proposes that the $\{223\}\langle 011\rangle$ orientation is the final stable orientation in plane strain compression. There is additional literature on cross-rolling of low carbon steel sheets [5], a strain state similar to plane strain along the transverse direction (PSTD). Results from Wronski et al. [5] show that the shear orientation and $\gamma$ fiber are stable orientations after near $80 \%$ thickness reduction.

There has been limited investigation into the texture evolution along other strain paths relevant for sheet metal forming. Yerra et al. [6] is one of the most complete, following five different strain paths from equal biaxial to uniaxial tension, all with the largest strain values in the rolling direction. The orientation distribution functions (ODFs) are measured at a single level of strain for each strain path. In Yerra et al. [6], the experimental textures were not found to change greatly between the different strain modes nor the volume fractions of specific orientations. However a calculation using the Lamel model by the same authors show a significant difference between the strain paths. Other notable works include Luzin et al. [15], which measured texture after balanced biaxial straining. The $\gamma$ fiber in a balanced biaxial strain mode is intensified, in agreement with Mirshams et al. [16] and theoretical calculations that predict the $\theta(\langle 100\rangle$ parallel to ND direction) and $\gamma$ fibers to be the stable orientations in uniaxial compression along the ND. Other strain modes, such as torsion [17] and simple shear [18] are outside the scope of this paper.

As steel is such a relevant industrial material, there have been attempts to predict the texture evolution. Savoie and Jonas [19] used a rate sensitive analysis to model the texture along a flange during deep drawing. These simulations include a rotation rate map that shows the stable end orientations for different constraints imposed on the models. They found that all initial orientations tend to rotate toward the $\{223\}\langle 110\rangle$ orientation. Toth et al. [20] used a rate dependent slip theory that contains models for pancake, lath and Taylor models to calculate texture evolution in low carbon steels during rolling. Starting with the 'RGoss' orientation, $\{111\}\langle 110\rangle$, and shear orientations; they found the $\{111\}\langle 112\rangle$ orientation also forms, but becomes unstable at higher strains. Many more stable orientations were found using this method, but most were observed along the $\alpha$ fiber and $\gamma$ fiber as well as $\{554\}\langle 225\rangle$. Raabe [21] used a Taylor type model to predict the rolling texture in low carbon deep drawing steel as a function of reduction ratio. The Taylor 
model showed increases in the intensity of the $\alpha$ fiber and $\gamma$ fiber in good agreement with experiment [21].

\section{Methods}

\section{Experimental}

\section{Chemical analysis}

Chemical analysis was performed following ASTM E415 [22] for all elements except sulfur which followed ASTM E1019 [23]. The resulting elemental mass fractions are given in Table 1. These are within the required parameters for AISI/SAE 1008 steel with the exception of manganese which is slightly lower than specified.

\section{Grain size measurements}

The average grain size was measured according to ASTM E112 [24]. Linear intercept methods along the rolling direction (RD), transverse direction (TD) and normal direction (ND) were performed along five lines in three images over two different samples for all six cross-sectional views ( $\mathrm{N}=60$ per direction). These averages were summed together to find the overall average grain size of $18.7 \mu \mathrm{m}$ and aspect ratios of 1.0:0.78:0.46 for RD:TD:ND, normalized to RD. Complete information on the grain size is shown in Table 2.

\section{Uniaxial sample testing}

Samples were rough cut from $1.17 \mathrm{~mm}$ (0.046 in, 18 gauge) thick sheet stock into sample blanks approximately $19 \mathrm{~mm}(0.75 \mathrm{in}) \times 200 \mathrm{~mm}(8 \mathrm{in})$ using a hydraulic shear. A TENSILKUT ${ }^{\mathrm{a}}$ machine was used to create dogbone samples that conform to ASTM E8 [25] standards for sheet specimens with a $50.8 \mathrm{~mm}$ (2 inch) gauge length and $12.7 \mathrm{~mm}$ (0.5 inch) width. After machining, samples were loaded into a screw driven tensile frame moving at a crosshead speed of $0.1 \frac{\mathrm{mm}}{\mathrm{sec}}$ resulting in a nominal strain rate of $0.002 \frac{\mathrm{strain}}{\mathrm{sec}}$. Axial and transverse strains were measured using extensometers. The strains imparted to the samples are listed in Table 3. The von Mises effective strain was calculated assuming volume conservation and neglecting shear strains:

$$
\epsilon_{\text {eff }}=\frac{\sqrt{2}}{3}\left[\left(\epsilon_{11}-\epsilon_{22}\right)^{2}+\left(\epsilon_{22}-\epsilon_{33}\right)^{2}+\left(\epsilon_{33}-\epsilon_{11}\right)^{2}\right]^{\frac{1}{2}}
$$

\section{Multi-axial sample testing}

Samples for non-uniaxial stain paths were loaded in a custom testing machine [26] using a sample geometry developed by Raghavan [27]. A clamp hold down force of $-450 \mathrm{kN}$ (force control) and a ram rate of $0.1 \frac{\mathrm{mm}}{\mathrm{sec}}$ was applied resulting in a nominal strain rate of $0.2 \frac{\mathrm{strain}}{\mathrm{sec}}$. Digital Image Correlation (DIC) was used to measure the plastic strains from a circular area with an undeformed diameter of $32 \mathrm{~mm}$ (1.25 inches). Strains are listed in Table 3.

Table 1 Mass fraction analysis performed by an independent lab per [22] and [23] for sulfur

\begin{tabular}{lll}
\hline Element & Measured & $\mathbf{1 0 0 8}$ AISI-SAE carbon steel grade [8] \\
\hline Carbon & 0.0007 & $0.0010 \mathrm{max}$ \\
Manganese & 0.0029 & 0.0030 to 0.0050 \\
Phosphorus & 0.00010 & $0.00040 \mathrm{max}$ \\
Sulfur & 0.00014 & $0.00040 \mathrm{max}$ \\
Iron & Balance & \\
\hline
\end{tabular}


Table 2 Average grain sizes for 1008 steel using the linear intercept method

\begin{tabular}{lccccc}
\hline Plane & Direction & $\begin{array}{c}\text { Average } \\
\text { grain size }[\boldsymbol{\mu m}]\end{array}$ & $\begin{array}{c}\text { Standard } \\
\text { deviation }[\boldsymbol{\mu m}]\end{array}$ & $\begin{array}{c}\text { Confidence } \\
\text { interval }[\boldsymbol{\mu m}]\end{array}$ & $\begin{array}{c}\text { Relative } \\
\text { accuracy [\%] }\end{array}$ \\
\hline ND & RD & 24.15 & 2.94 & 1.10 & 4.55 \\
TD & RD & 28.72 & 3.25 & 1.21 & 4.22 \\
\hline ND & TD & 17.85 & 1.50 & 0.56 & 3.14 \\
RD & TD & 23.40 & 2.27 & 0.85 & 3.62 \\
\hline RD & ND & 11.79 & 0.93 & 0.35 & 2.96 \\
TD & ND & 12.59 & 0.78 & 0.29 & 2.32 \\
\hline
\end{tabular}

Images were recorded after etching with Nital (2\%) for 45 seconds. Images were recorded at $200 \times$ magnification on all three planes on two different samples each. $n=30$ for each row.

\section{Texture measurements}

Crystallographic texture measurements were performed using neutron diffraction at the BT-8 Residual Stress Diffractometer at NIST [28]. Strained and as received samples were cut into $8 \mathrm{~mm}$ squares and stacked and glued together until a cube was formed. For the average grain sizes calculated in Table 2, there are on the order of $2 \times 10^{7}$ grains in each sample. Three complete pole figures from the (200), (220), and (211) reflections were measured. A $5^{\circ}$ hexagonal grid [29] of the pole figure space was used, resulting in 469 separate measurements, with the exception of the URD and UTD samples strained to $10 \%$ and $20 \%$. In these four samples a hexagonal grid of $6^{\circ}$ spacing was used, resulting in 331 separate measurements. The raw data was then processed in the program PF [30] and converted to pole figures in the popLA format [31]. The as received samples exhibited orthotropic

Table 3 Applied strains, measured after unloading the samples, expressed as true strain

\begin{tabular}{|c|c|c|c|c|c|}
\hline Name & Nominal strain (\%) & $\epsilon_{\mathrm{RD}}$ & $\epsilon_{\mathrm{TD}}$ & $\epsilon_{\mathrm{ND}}$ & $\epsilon_{\text {eff }}$ \\
\hline URD10 & $10 \%$ & 0.100 & -0.063 & -0.037 & 0.101 \\
\hline URD20 & $20 \%$ & 0.200 & -0.127 & -0.072 & 0.202 \\
\hline URD30 & $30 \%$ & 0.302 & -0.186 & -0.116 & 0.305 \\
\hline PSRD10 & $10 \%$ & 0.10 & -0.01 & -0.09 & 0.11 \\
\hline PSRD10 & $10 \%$ & 0.10 & -0.01 & -0.09 & 0.11 \\
\hline PSRD20 & $20 \%$ & 0.20 & -0.02 & -0.17 & 0.21 \\
\hline PSRD20 & $20 \%$ & 0.20 & -0.02 & -0.17 & 0.21 \\
\hline PSRD30 & $30 \%$ & 0.33 & -0.03 & -0.29 & 0.36 \\
\hline PSRD30 & $30 \%$ & 0.33 & -0.03 & -0.30 & 0.37 \\
\hline BB10 & $10 \%$ & 0.10 & 0.10 & -0.19 & 0.19 \\
\hline BB20 & $20 \%$ & 0.20 & 0.20 & -0.40 & 0.41 \\
\hline BB30 & $30 \%$ & 0.32 & 0.32 & -0.63 & 0.63 \\
\hline PSTD10 & $10 \%$ & -0.01 & 0.10 & -0.09 & 0.11 \\
\hline PSTD10 & $10 \%$ & -0.01 & 0.10 & -0.09 & 0.11 \\
\hline PSTD20 & $20 \%$ & -0.02 & 0.22 & -0.20 & 0.24 \\
\hline PSTD20 & $20 \%$ & -0.02 & 0.22 & -0.20 & 0.24 \\
\hline PSTD30 & $30 \%$ & -0.03 & 0.34 & -0.31 & 0.37 \\
\hline PSTD30 & $30 \%$ & -0.03 & 0.33 & -0.30 & 0.37 \\
\hline UTD10 & $10 \%$ & -0.063 & 0.100 & -0.037 & 0.101 \\
\hline UTD20 & $20 \%$ & -0.125 & 0.201 & -0.076 & 0.203 \\
\hline UTD30 & $30 \%$ & -0.167 & 0.297 & -0.130 & 0.297 \\
\hline
\end{tabular}

For uniaxial testing, strains are measured along the RD and TD by extensometers, for BB and PS testing RD and TD strains are measured by digital image correlation. ND strains are calculated from volume conservation. The von Mises effective strain is calculated as shown in equation 1. 
sample symmetry from the rolling process. All sample strains occurred aligned with the initial orthotropic symmetry resulting in orthotropic symmetry in the strained samples. For these reasons, orthotropic sample symmetry was applied to the pole figures and the orientation distribution functions (ODFs).

\section{Orientation Distribution Function (ODF) calculation}

From the series of pole figures, orientation distribution functions (ODFs) were calculated to determine the distribution of grains as a function of crystallographic orientation in the bulk material. Two different ODF solvers were investigated, the WIMV method [32] in popLA and mTex [33]. The calculation of the ODF is an inverse problem, which are known to not necessarily have a unique solution. For each solver a range of different ODFs can be calculated that match the pole figures depending on the, number of iterations of the ODF solving loop, resolution of the ODF, and other input parameters. To facilitate comparison with the VPSC ODF calculations, the WIMV method was used. Uncertainty in ODF measurements is an ongoing measurement challenge currently under investigation [34]. For the purposes of this paper, an uncertainty of $10 \%$ in the ODF values is assumed [35].

Good agreement ( $2 \%$ calculation error) with the pole figures was found after 12 iterations. Experimental and recalculated pole figures were qualitatively inspected to check agreement as well. The ODF from the as received sample was used to calculate an input file for the VPSC analysis. The ODF is expressed in a crystal orientation distribution file that has a regular $5^{\circ}$ grid in the Bunge Euler angles $\phi_{1}, \Phi, \phi_{2}$. Due to the crystal and sample symmetries, the ODF can be expressed in a subspace with the range $0^{\circ} \leq \phi_{1} \leq 90^{\circ}$, $0^{\circ} \leq \Phi \leq 90^{\circ}, 0^{\circ} \leq \phi_{2} \leq 90^{\circ}$, albeit with an additional three fold symmetry in this subspace. A value for intensity is listed at each point in this grid, expressed in units of 'multiples of a uniform distribution' or MUD (often referred to as 'multiples of a random distribution' MRD).

Excerpts from the ODF were also extracted along fiber textures. The fibers of interest for this paper are the $\alpha$ fiber, $\gamma$ fiber and $\theta$ fiber. The $\alpha$ fiber runs along the $\Phi$ axis, with $\phi_{1}=0^{\circ}$ and $\phi_{2}=45^{\circ}$. The $\gamma$ fiber runs along the $\phi_{1}$ axis, with $\Phi=55.4^{\circ}$ and $\phi_{2}=45^{\circ}$. The $\gamma_{1}, \gamma_{3}$ and $\gamma_{2}, \gamma_{4}$ pairs are equivalent points by symmetry.The $\theta$ fiber runs along the $\phi_{1}$ axis, with $\Phi=0^{\circ}$ and $\phi_{2}=45^{\circ}$. Specific orientations of interest are listed in Table 4, with a shorthand name, Miller indices, and Bunge Euler angles. Additionally, difference ODF plots were created between adjacent strain steps. These difference ODFs show the locations that grains are rotating to or away from. However, as a consequence of the nonlinear nature of the intensity scale of the ODF, caution should be applied to ascribing quantitative significance to the values in the difference plots. To aid in the idenification of the orientations listed in Table 4, a key showing the location of each orientation located in the $\phi_{2}=45^{\circ}$ cross section is shown in Figure 1 .

\section{Computational}

The viscoplastic self-consistent (VPSC) model developed by Lebensohn and Tomé [36] was used to simulate the texture development. The VPSC model treats each grain as a viscoplastic ellipsoidal inclusion embedded in and interacting with a homogeneous effective matrix (HEM). Under the HEM assumption, individual grain response varies as a function of its orientation and the stiffness of the grain-to-matrix interaction, depending on 
Table 4 Relevant orientations investigated

\begin{tabular}{|c|c|c|c|c|c|}
\hline Name & $\{h k l\}$ & $\langle u v w\rangle$ & $\phi_{1}$ & $\Phi$ & $\phi_{2}$ \\
\hline Cube & $\{001\}$ & $\langle 100\rangle$ & $45^{\circ}$ & $0^{\circ}$ & $45^{\circ}$ \\
\hline Shear & $\{001\}$ & $\langle 1 \overline{1} 0\rangle$ & $0^{\circ}$ & $0^{\circ}$ & $45^{\circ}$ \\
\hline Goss & $\{011\}$ & $\langle 100\rangle$ & $90^{\circ}$ & $90^{\circ}$ & $45^{\circ}$ \\
\hline RGoss & $\{011\}$ & $\langle 0 \overline{1} 1\rangle$ & $0^{\circ}$ & $90^{\circ}$ & $45^{\circ}$ \\
\hline Copper & $\{112\}$ & $\langle\overline{1} \overline{1} 1\rangle$ & $90^{\circ}$ & $35.26^{\circ}$ & $45^{\circ}$ \\
\hline Brass & $\{011\}$ & $\langle 2 \overline{1} 1\rangle$ & $54.74^{\circ}$ & $90^{\circ}$ & $45^{\circ}$ \\
\hline S & $\{213\}$ & $\langle\overline{3} \bar{\sigma} 4\rangle$ & $58.98^{\circ}$ & $36.70^{\circ}$ & $63.44^{\circ}$ \\
\hline$\{554\}$ & $\{554\}$ & $\langle\overline{2} \overline{2} 5\rangle$ & $90^{\circ}$ & $60.50^{\circ}$ & $45^{\circ}$ \\
\hline$\gamma_{1}$ & $\{111\}$ & $\langle 1 \overline{1} 0\rangle$ & $0^{\circ}$ & $54.74^{\circ}$ & $45^{\circ}$ \\
\hline$\gamma_{2}$ & $\{111\}$ & $\langle 1 \overline{2} 1\rangle$ & $30^{\circ}$ & $54.74^{\circ}$ & $45^{\circ}$ \\
\hline$\gamma_{3}$ & $\{111\}$ & $\langle 0 \overline{1} 1\rangle$ & $60^{\circ}$ & $54.74^{\circ}$ & $45^{\circ}$ \\
\hline$\gamma_{4}$ & $\{111\}$ & $\langle\overline{1} \overline{1} 2\rangle$ & $90^{\circ}$ & $54.74^{\circ}$ & $45^{\circ}$ \\
\hline$\alpha_{1}$ & $\{115\}$ & $\langle 1 \overline{1} 0\rangle$ & $0^{\circ}$ & $15.79^{\circ}$ & $45^{\circ}$ \\
\hline$\alpha_{2}$ & $\{113\}$ & $\langle 1 \overline{1} 0\rangle$ & $0^{\circ}$ & $25.24^{\circ}$ & $45^{\circ}$ \\
\hline$\alpha_{3}$ & $\{112\}$ & $\langle 1 \overline{1} 0\rangle$ & $0^{\circ}$ & $35.26^{\circ}$ & $45^{\circ}$ \\
\hline$\alpha_{4}$ & $\{223\}$ & $\langle 1 \overline{1} 0\rangle$ & $0^{\circ}$ & $43.31^{\circ}$ & $45^{\circ}$ \\
\hline
\end{tabular}

All orientations are described by Miller Indices and Bunge Euler angles.

the choice of homogenization methods at grain level. Details of the model can be found in [36].

A file containing 24938 discrete orientations was created to approximate the calculated ODF from the as received sample. This list of orientations was used as an input file to the VPSC algorithm. Three other input files were also required: a file containing the applied velocity gradients and applied Cauchy stresses; a file containing the unit cell parameters, elastic moduli of the single crystal, slip planes, and hardening behavior of slip families; and an input file containing the path and name of the subfiles, grain aspect ratio, hardening model, and interaction model.

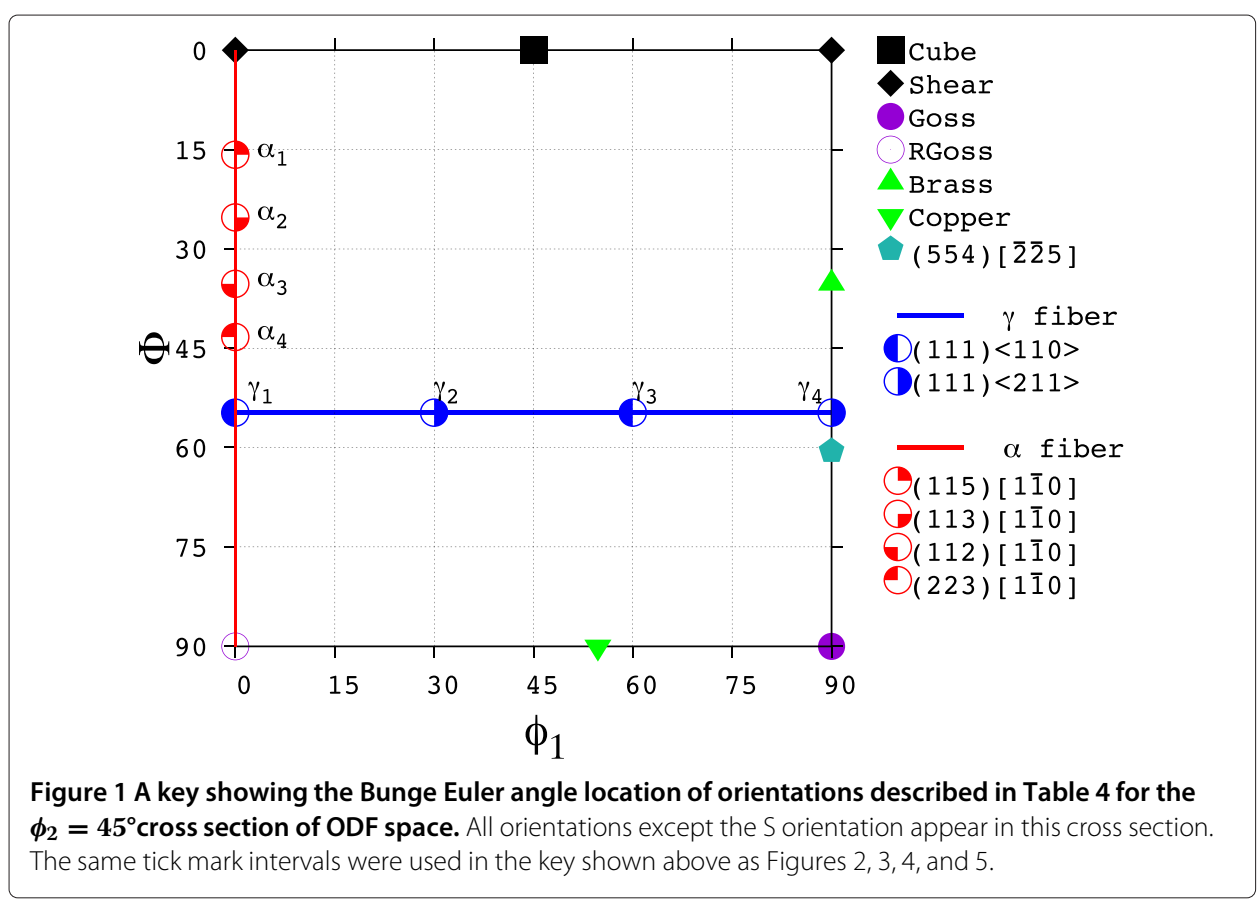


For all simulations a velocity gradient is imposed on all components of the velocity gradient tensor matching the macroscopic strains in Table 3 in the following manner. Imposed velocity tensors were the $\epsilon_{\mathrm{RD}}, \epsilon_{\mathrm{TD}}$ and $\epsilon_{\mathrm{ND}}$ strains normalized such that the highest positive strain was set equal to 1 . The strain increment was the value of strain along the highest positive strain direction. All stresses calculated by the VPSC model are therefore deviatoric stresses. Only the $\{110\}\langle 111\rangle$ and $\{112\}\langle 111\rangle$ families of slip planes and directions were considered in this model as initial experimentation including the $\{123\}\langle 111\rangle$ family of planes did not show a significant effect on the texture. The ellipsoid ratios used were based on the experimental data listed in Table 2 normalized along the rolling direction and having values of 1.0, 0.78 and 0.46 for the RD, TD and ND respectively. Secant linearization and first nearest neighbor interaction models were chosen. The outputs used here from the VPSC algorithm include a list of discrete orientations that have been rotated to new orientations due to the imposed velocity gradient. These discrete orientations were binned into a regular grid to create an ODF.

\section{Results}

\section{Experimental textures}

Three different techniques have been used to show how the experimentally measured texture evolves as a function of applied strain. Figure 2 shows the intensities of the ODF

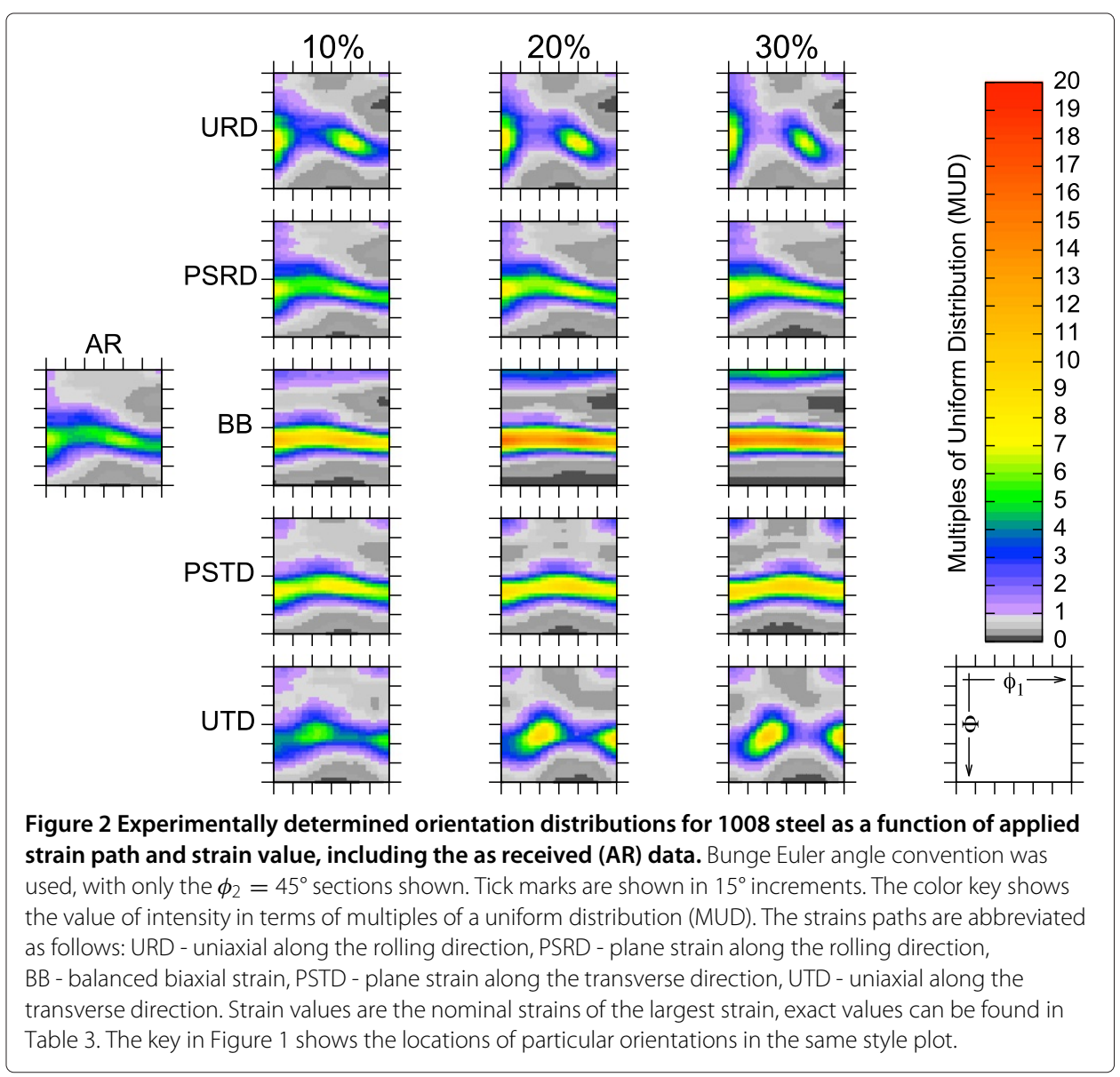



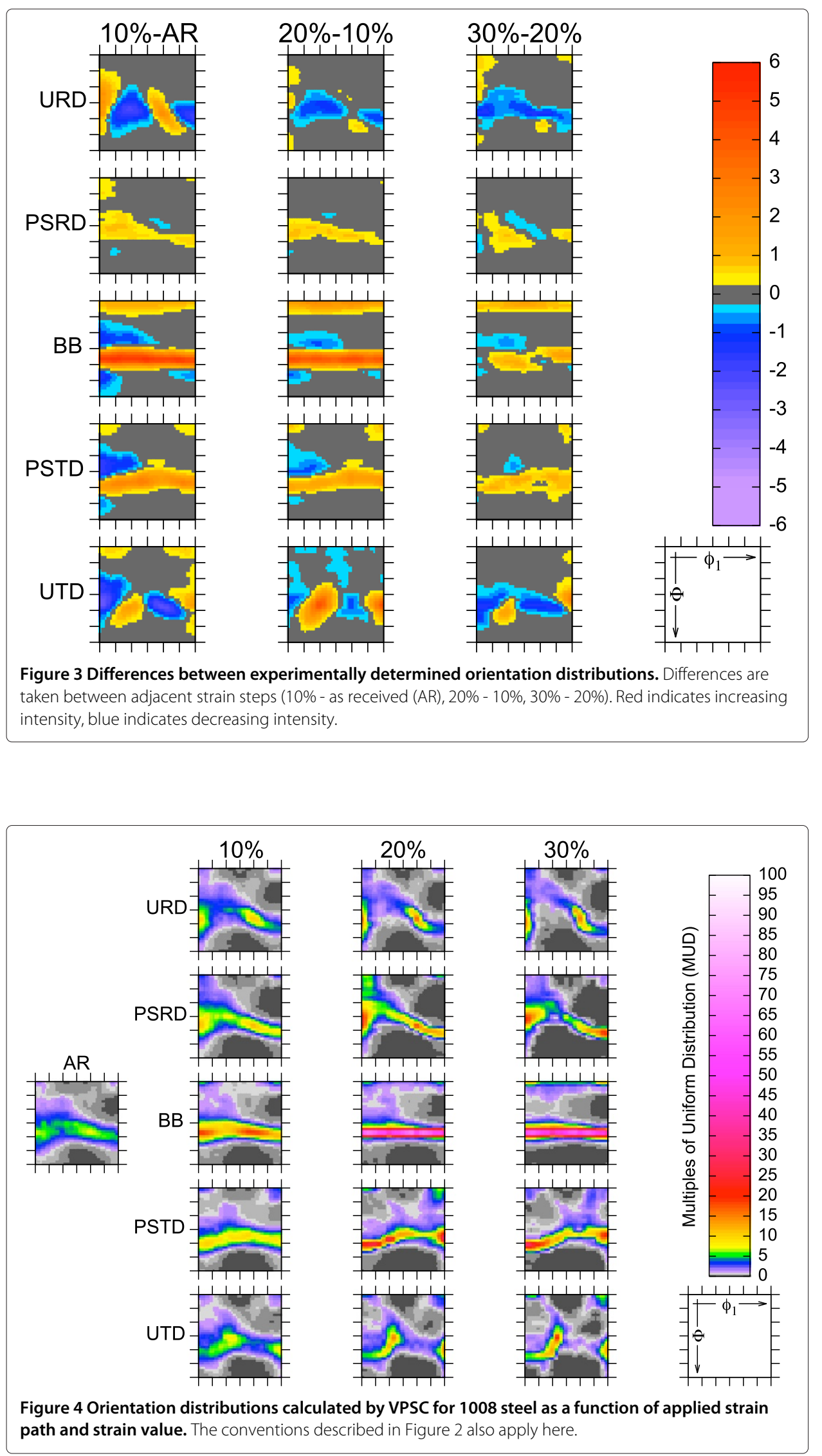


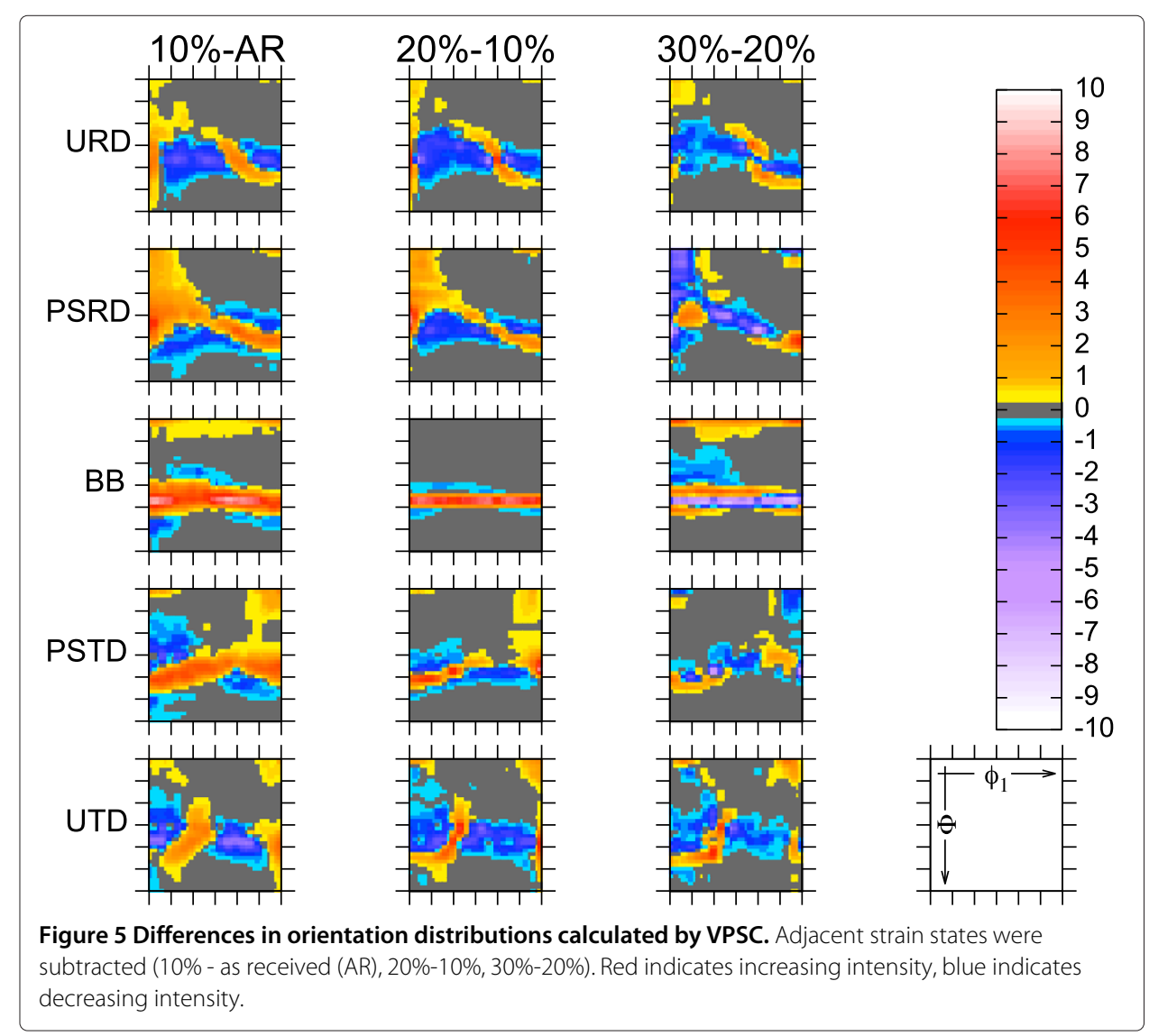

on $\phi_{2}=45^{\circ}$ cross sections. Figure 3 is a plot of the differences between adjacent strain steps $(10 \%$ - as received, $20 \%-10 \%, 30 \%-20 \%)$ on the same $\phi_{2}=45^{\circ}$ cross sections shown in Figure 2. The smaller strain step is subtracted from the larger strain step, so positive values indicate an increase in intensity at the given orientation. The third technique extracts line profiles along a path with two fixed Euler angles. These line profiles correspond to the $\alpha$ fiber (Figure 6 top), $\gamma$ fiber (Figure 7 top), and $\theta$ fiber (Figure 8 top). For brevity, the discussion of the URD deformation contains the relevant figure numbers for discussion of the fiber components, they will not be repeated for each strain mode.

Figure 2 includes the as received texture, as well as the three nominal strain increments along the five strain paths explored. The as received texture of the 1008 steel is typical for a rolled body centered cubic (BCC) material for deep drawing applications, with strong intensity along the $\gamma$ fiber, and peaks along the $\langle 110\rangle$ RD directions of the (111) plane. The intensity values for $\alpha$ fiber orientations and the shear orientation are also larger than in a uniform texture distribution. The intensities of cube, shear, Goss, RGoss, copper, brass, and $\mathrm{S}$ orientations are low, as expected in a $\mathrm{BCC}$ material.

When samples are strained in uniaxial tension along the rolling direction (URD), the ODF intensity along the $\gamma_{1}$ and $\gamma_{3}$ orientations increases, as shown in the first row in Figure 2, Figure 3, and Figure 7. The ODF intensity along the $\{554\}\langle\overline{2} \overline{2} 5\rangle, \gamma_{2}$, and $\gamma_{4}$ decrease. The largest change in the ODF intensities are along these orientations occurs while straining from $0 \%$ to $10 \%$. The ODF intensity along all of the $\alpha$ fiber orientations 


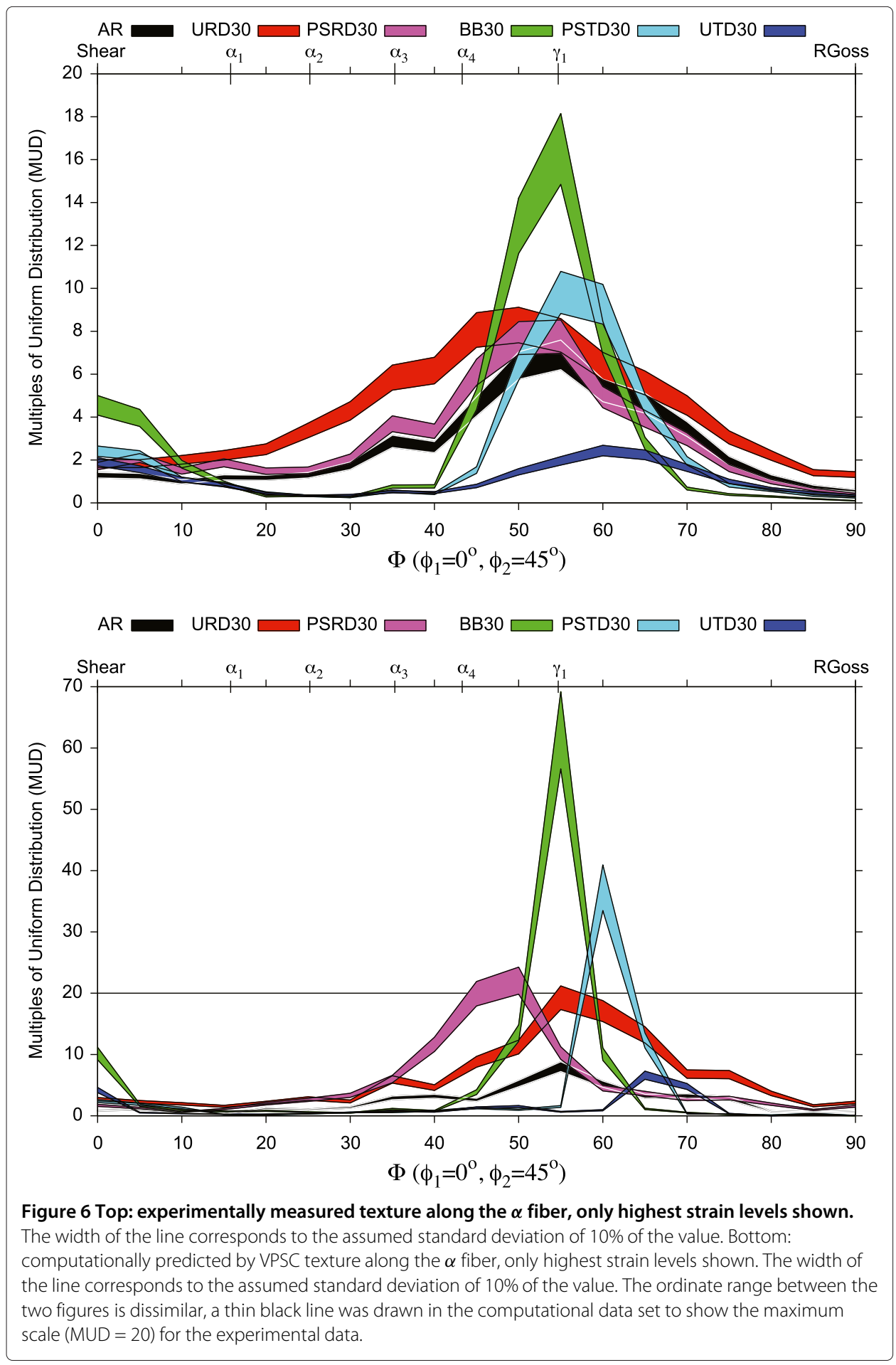

increase, shown in Figure 2, Figure 3, and Figure 6. Along the $\theta$ fiber the grains along the shear orientation increase, shown in Figure 2, Figure 3, and Figure 8, but the ODF intensity along the cube orientation remains the same.

The PSRD strain state is notable in that there are very few changes from the as received structure, depicted in the second row in Figures 2 and 3. For all of the orientations 


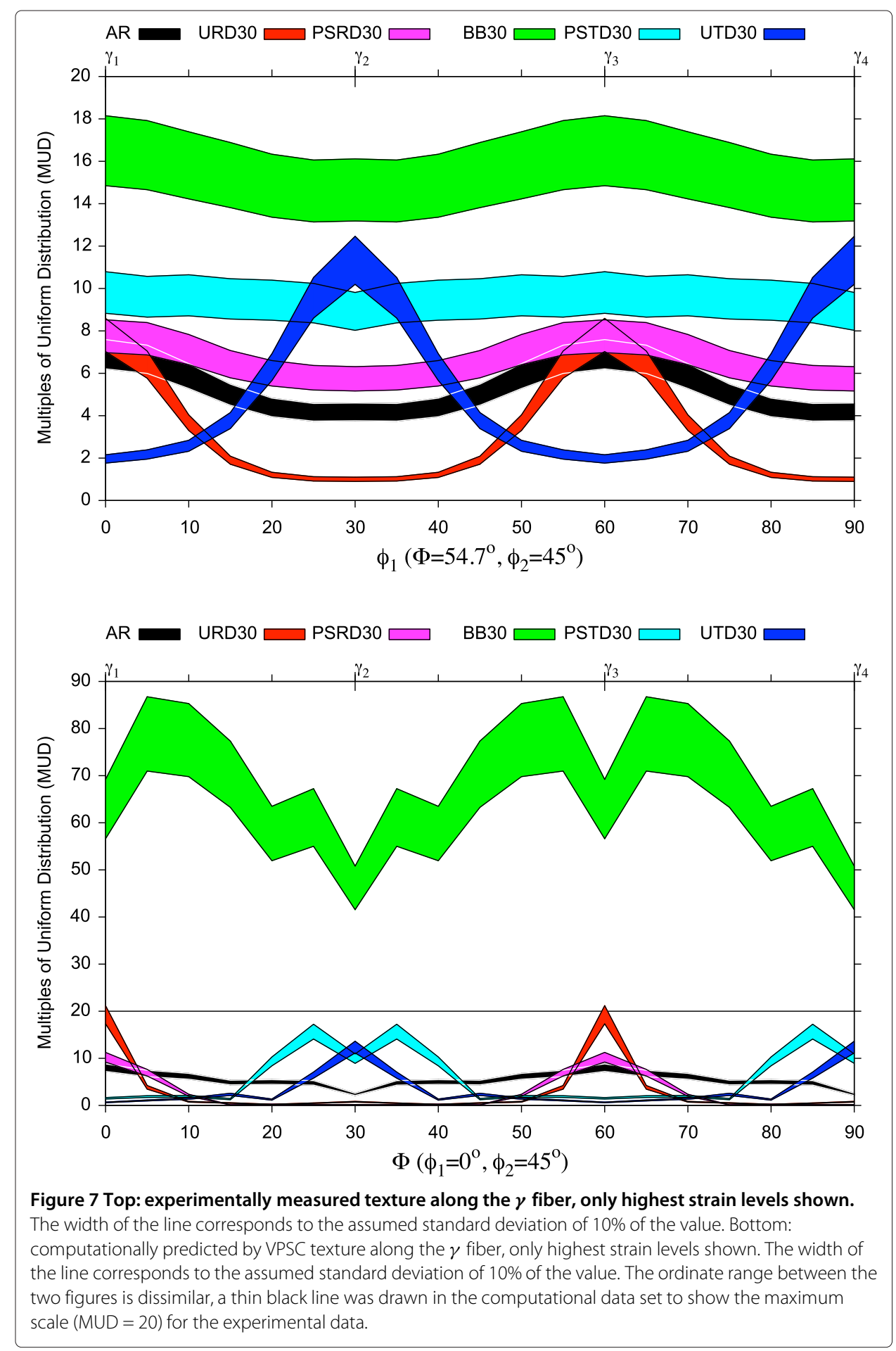

investigated, the ODF intensity changes only very slightly at each strain level. The ODF intensity of orientations along the $\alpha$ fiber are basically unchanged. There are some modest increases in the intensity along the $\gamma$ fiber, particularly filling in the $\gamma_{2}$ and $\gamma_{4}$ orientations to make the intensity along the $\gamma$ fiber slightly higher and more uniform in intensity. The $\theta$ fiber shows the same trend as the samples deformed with uniaxial tension 


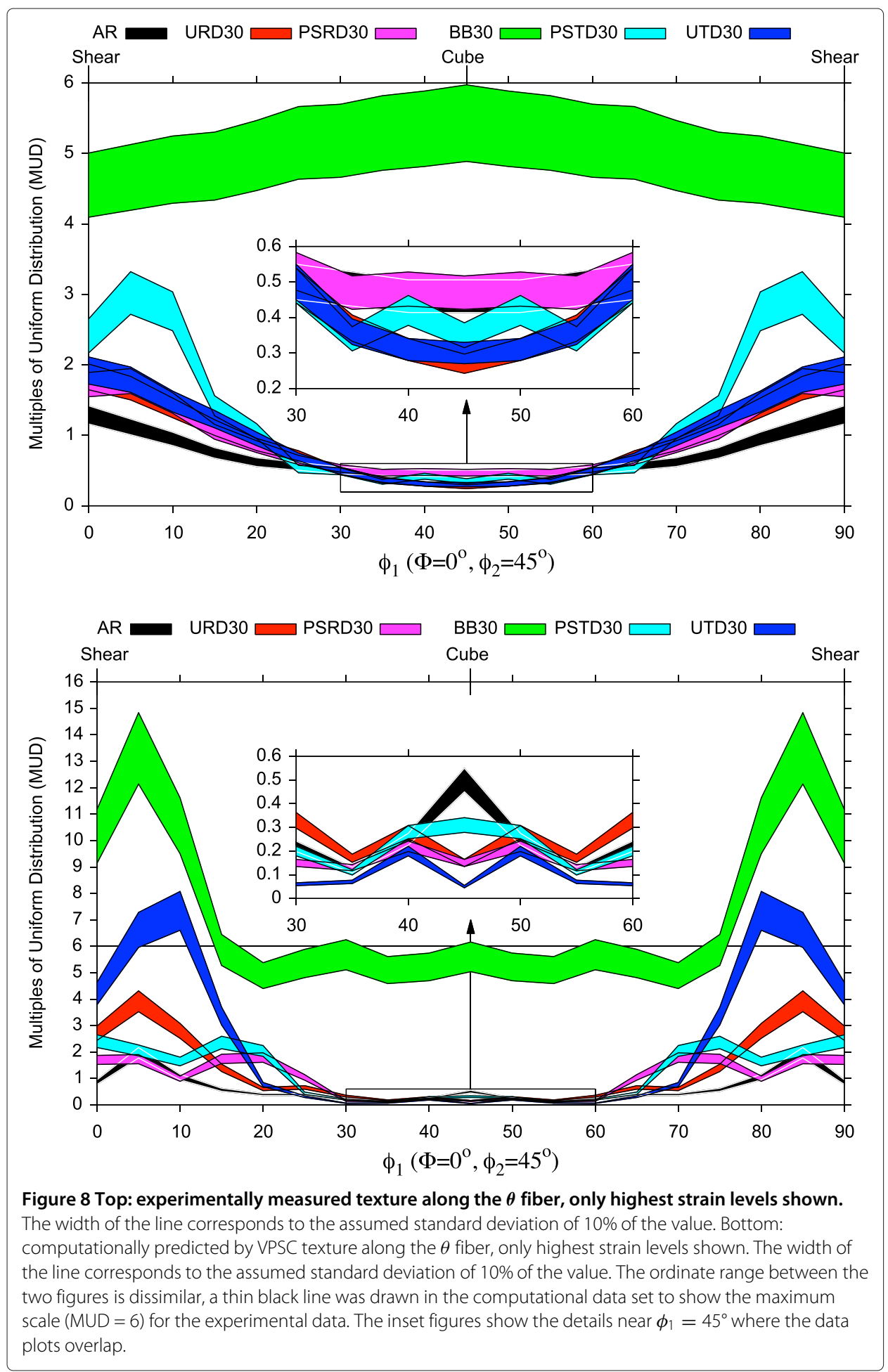

along the rolling direction with the shear orientation increasing and the cube orientation unchanged.

The dominant texture evolution in the BB strain state is intensification of the $\gamma$ fiber. The $20 \%$ sample still shows the highest intensity along the $\langle 110\rangle$ RD directions, but by $30 \%$ strain all orientations along the $\gamma$ fiber are approximately equal and have an intensity 
of 15 to 16 times higher than a uniform distribution. Grains oriented along the $\alpha$ fiber have decreased sharply outside the range of the $\gamma$ fiber and $\theta$ fiber. The BB strain state also shows the strongest changes along the $\theta$ fiber. The cube orientation increases from a value of 0.5 MUD in the as received strain state to over 5 MUD after 30\% BB straining. Similarly, the shear orientation increases dramatically.

The PSTD strain state results in a texture evolution that is similar to the BB strain state. Grains oriented along the $\gamma$ fiber increase, particularly the $\gamma_{2}$ and $\gamma_{4}$ orientations, to become nearly constant in intensity at 10 MUD by $30 \%$ strain. Grains oriented along the $\alpha$ fiber decrease, except for those near the $\gamma$ fiber. The ODF intensity along the lower part of the $\alpha$ fiber $\left(\phi_{1} \geq 60^{\circ}\right)$ increases more than observed in the RD, PSRD or BB strain states. There is an increase in the number of grains oriented along the shear orientation, while the cube orientation remains unchanged.

Due to the symmetries in this projection of Euler space, the texture evolution of UTD samples look like the corresponding URD ODF mirrored about the $\phi_{1}=90^{\circ}$ axis. Grains oriented along the $\gamma_{2}$ and $\gamma_{4}$ orientations increase, while $\gamma_{1}$ and $\gamma_{3}$ intensity decreases. Orientations along the $\alpha$ fiber decrease. There is some increase in the copper orientation, which can be related by symmetry operations to an orientation near the $\alpha_{3}$ orientation. Along the $\theta$ fiber the fraction of grains oriented along these directions are similar to measurements of the URD and PSRD samples.

\section{Computational textures}

Similar to the experimental data shown in Figure 2, Figure 4 shows the calculated intensities of the ODF in $\phi_{2}=45^{\circ}$ cross sections from VPSC simulations. Figure 5 is a plot of the differences between adjacent strain steps (10\% - as received, $20 \%-10 \%, 30 \%-20 \%)$ in the ODFs calculated with VPSC. Line profiles along the $\alpha$ fiber (Figure 6 bottom), $\gamma$ fiber (Figure 7 bottom) and $\theta$ fiber (Figure 8 bottom) are also shown. Similar to the experimental data, the discussion of the URD deformation contains the relevant figure numbers for discussion of the fiber components, they will not be repeated for each strain mode.

The intensity scale for each of the computational figures was adjusted to accommodate the higher intensity values calculated in the VPSC code. However the color scale in the range of the experimental intensities are unchanged for Figure 4 and Figure 5 to allow for comparison, additional colors were added for intensities outside of the experimental range. For Figures 6, 7, and 8 a thin black line is used to depict the maximum in the experimental line profile figures.

The VPSC as received texture shown in Figure 4 is similar to, but less smooth than the experimental as received texture shown in Figure 2. Regions around $\gamma_{2}$ and $\gamma_{4}$ are slightly depleted in intensity compared to the experimental data. Similarly, areas around the copper and brass orientations are also lower than observed experimentally.

The main intensity changes for URD occur along $\gamma_{1}$ and $\gamma_{3}$. There are corresponding decreases in the $\gamma_{2}$ and $\gamma_{4}$ orientations. As the amount of strain increases, the intensity around the $\gamma_{3}$ orientation increases in a very narrow band in the $\phi_{1}$ axis while broadening in the $\Phi$ axis at higher strains. The $\alpha$ fiber increases from the as received sample, but with larger increases at $\Phi>55^{\circ}$ than at $\Phi<55^{\circ}$ observed in the experimental data. Along the $\theta$ fiber, the shear orientation shows a sharp drop, observed in all VPSC data sets. These observations are likely computational artifacts due to the low number of grains $\left(2.5 \times 10^{5}\right)$ compared to the experimental data $\left(\approx 2 \times 10^{7}\right)$. The $\theta$ fiber 
orientations show modest increases in the shear texture, but the cube texture is essentially unchanged.

When the strain state changes to plane strain along the rolling direction (PSRD), the texture evolution is similar to the VPSC URD sample. The $\gamma_{1}$ through $\gamma_{3}$ orientations increase but the $\gamma_{2}$ and $\gamma_{4}$ orientations decrease. However, the $\{554\}$ orientation, which is close to the $\gamma_{4}$ orientation, increases like the $\gamma_{1}$ and $\gamma_{3}$ orientations. Unlike experimental data, orientations near the $\alpha_{3}$ and $\alpha_{4}$ orientations show large increases, particularly at 20\% strain, but the other $\alpha$ fiber orientations only show slight increases in ODF intensity. Similar to the $\alpha_{4}$ orientations, the shear orientation shows a large increase at $20 \%$ strain, and then decreases back to the as received intensity at $30 \%$. The cube and shear orientations in the $\theta$ fiber are also largely unchanged.

Balanced biaxial (BB) strains show the sharpest texture evolution. The texture sharpening is primarily along the $\gamma$ fiber. All orientations along $\gamma$ fiber increase by similar amounts for each strain step, such that by 30\% strain, intensities of 80 MUD are observed. The $\alpha$ fiber shows a sharp peak where it intersects with the $\gamma$ fiber, but decreases in all other orientations. The $\theta$ fiber shows a large increase as well, predominantly along the shear orientation.

Plane strain along the transverse direction (PSTD) shows increases in the ODF intensity at all orientations along the $\gamma$ fiber after $10 \%$ strain, and then increases in the $\gamma_{2}$ and $\gamma_{4}$ thereafter. Similar to the URD sample, the $\alpha$ fiber increases more at $\Phi>55^{\circ}$ than at $\Phi<55^{\circ}$. The shear orientation increases in intensity, but the cube orientation remains nearly constant.

Finally, the UTD samples show intensity increases along $\gamma_{2}$ and $\gamma_{4}$, the $\langle 110\rangle$ TD directions of the (111) plane, but of lower magnitude than the PSTD strain state. The intensity of orientations $\gamma_{1}$ and $\gamma_{3}$ decrease. Like the URD case, as strain increases the highest intensity bands tend to align themselves with the $\Phi$ axis. The intensity along the $\alpha$ fiber orientations generally decrease, with only a slight increase at $\Phi=65^{\circ}$ to $70^{\circ}$. Orientations near the shear texture also show large increases.

\section{Discussion}

Qualitatively there is good agreement between the experimental and computational texture evolution models. Most changes from the texture evolution have the correct 'sense' to them, experimental increases are matched to computational increases and vice versa. Both the computational and experimental textures show significant changes even at relatively low (10\%) values of strain. There are some subtle differences between the Experimental and VPSC as received textures. As discussed in the results and methods sections, the VPSC as received texture is less smooth, likely due to the three order of magnitude reduction in the number of grains explored in the VPSC model (24 938 orientations) than in the experimental data (estimated at $\approx 2 \times 10^{7}$ ). For orientations with $\phi_{2}=45^{\circ}$ and $\Phi<20^{\circ}$ there are less than 10 grains for each of the orientations in the $5^{\circ}$ bin and at $\Phi=0$ most of the orientations only have 1 grain associated with each bin. This may also be the cause of oddities around the shear orientation in Figure 8, where large peaks are observed $5^{\circ}$ from the orientation that are not observed experimentally in Figure 8.

There are a few places where there is qualitative disagreement between the trends in the VPSC predictions and experimental data. In the experimental URD and PSRD strain states there are broad increases in the $\alpha$ fiber, particularly at $\Phi<55^{\circ}$, shown in Figure 6 . 
The VPSC data in Figure 6 shows some increase in the intensity at $\Phi<55^{\circ}$, but a larger increase in intensity at $\Phi>55^{\circ}$. Similarly the increase in cube orientation over the shear orientation observed experimentally in Figure 8 under BB straining was not observed in the VPSC predictions in Figure 8. At higher strain levels the VPSC model seemed to rotate grains away from the $\gamma$ fiber to orientations $\pm 5^{\circ}$ to $\pm 10^{\circ}$ away from $\Phi=55^{\circ}$ ( $\gamma$ fiber), as shown by the decreases in intensity along the $\gamma$ fiber in the difference map in Figure 5. This may also affect the PSTD results data, where the intensity pattern observed experimentally in Figure 7 does not match the VPSC predictions shown in Figure 7.

Quantitatively, the VPSC model tends to over predict the rate of texture evolution leading to much higher intensities and sharper orientation distributions. Overall, the intensity values for experimental data at 30\% strain better match the VPSC data at 10\% strain. The BB $30 \%$ strain state is a particularly good example of the difference in rate of texture evolution, with the intensity predicted by the VPSC over-predicted by a factor of 5 . Another example is the PSRD strain state, where the $\alpha_{4}$ and $\{554\}$ orientations are predicted to have increases larger than are observed experimentally.

Comparing this data with prior computational and analytical work, the uniaxial samples show the $\langle 110\rangle$ slip directions of the $\{111\}$ plane as stable orientations, and instability of slip directions $\langle 211\rangle$ in the $\{111\}$ plane. The PSRD strain state is indeed similar to the predictions for cold rolling the ferrite phase. The experimental data indicates that while the $\alpha_{4}(\{223\}\langle 1 \overline{1} 0\rangle)$ orientation is the most stable, there seems to be a broad distribution of orientations that are increasing at the strain levels investigated. The stability of the $\alpha$ fiber changes with more transverse direction strain, such that in the BB and PSTD strain states this fiber is not as stable. This result could be used in production, for applications where $\alpha$ fiber is not desirable, as more lubrication on the rollers or cross rolling could be used to remove some of the $\alpha$ fiber and increase the number of grains with orientations aligned with the $\gamma$ fiber; albeit with some increase in the cube and shear components as well. The increase in $\gamma$ fiber agrees well with observations by [15] and [16] but in our work more cube orientated grains are observed than by Luzin et al. [15]. The experimental and VPSC data are more consistent with the Lamel predictions than experimental data from Yerra et al. [6].

A similar study 5754 aluminum compared Los Alamos polycrystal plasticity (LApp), VPSC and visco-plastic fast Fourier transform (VPFFT) models for multiaxial strain states [37]. Hu et al. [37] showed similar trends to those observed here, with the rate of texture evolution over predicted in the VPSC model, particularly for the BB strain state. The VPFFT model calculated a rate of texture evolution closer to those observed experimentally. In the VPFFT model, the inter-grain and intra-grain interactions are included in the model, likely improving the accuracy. However, the VPFFT model described by Hu et al. [37] is discretized as voxel elements. Approximately 500 voxels were required for each grain, as opposed to the single ellipsoid per grain in the VPSC model, resulting in the VPFFT model containing one fifth as many grains as the VPSC model for a similar computational time. The reduction in number of grains resulted in a lower fidelity to the as received texture. The texture in the 5754 aluminum studied was much closer to a uniform distribution than the steel texture measured here, so a larger number of grains would be required to accurately model the steel microstructure. Following work by Dawson et al. [38], the addition of the elastic component to the viscoplastic models (EVPSC or EVPFFT approaches) is not expected to improve the model as the strains in this study are 
relatively large strains along monotonically increasing strain paths. Additionally, the construction of an accurate microstructure with respect to the spatial correlation of adjacent grains required as an input to a VPFFT or crystal plasticity based finite element analysis (CP-FEA) models is a topic of current research.

However, there is some concern that even accurate microstructure may be insufficient to model the deformation of polycrystalline materials. Pokharel et al. [39] compare a modeled microstructure in VPFFT that is taken from experimental data from near field high-energy X-ray diffraction microscopy (nf-HEDM) [40]. Even with a microstructure model that closely matches the experimental data, the agreement between experiment and model are not as close as desired. Similar to the results shown in this paper, there is reasonable qualitative agreement, but there are significant qualitative and quantitative variations. Pokharel et al. [39] conclude that the "empirically derived constitutive relations used in continuum scale simulations" (i. e. the Voce model in VPFFT and VPSC) as the likely cause of the disagreement and recommend further work on the development of a constitutive relation based on dislocation interactions. Pokharel et al. [39] also touches on CP-FEA, but states that the empirically derived continuum level constitutive relationships used in FEA may suffer similar problems as observed in the VPFFT model.

\section{Conclusions}

To summarize, the crystallographic texture evolution in a 1008 low carbon steel along five different strain states have been experimentally measured and computationally predicted using VPSC. Comparisons between measured texture evolution and computational texture evolution indicates that the VPSC model is generally qualitatively accurate in predicting the orientations to which grains rotate. Quantitatively, the VPSC model over predicts the rate at which grains will rotate, particularly in multi-axial strain states such as balanced biaxial.

\section{Endnote}

${ }^{\text {a }}$ Certain commercial equipment, instruments, or materials are identified in this paper in order to specify the experimental procedure adequately. Such identification is not intended to imply recommendation or endorsement by the National Institute of Standards and Technology, nor is it intended to imply that the materials or equipment identified are necessarily the best available for the purpose.

Competing interests

The authors declare that they have no competing interests.

\section{Authors' contributions}

AC calculated the ODFs, compared the data and lead writing of the manuscript; LH conducted the VPSC simulations; TGH measured and analyzed the experimental pole figures; ADR advised LH in VPSC, reviewed our work and assisted in interpretation. All authors read and approved the final manuscript.

\section{Acknowledgements}

The authors thank Steve Banovic, Tim Foecke, Mark ladicola and Mark Stoudt at the NIST Center for Metal Forming for advice and assistance, and the Research Associate Program at the National Academies for supporting this work.

\section{Author details}

${ }^{1}$ National Institute of Standards and Technology, 100 Bureau Dr., 20899, Gaithersburg, MD, USA. ${ }^{2}$ Carnegie Mellon University, 5000 Forbes Avenue, 15213, Pittsburgh, PA, USA. ${ }^{3}$ IBM Semiconductor Research and Development Center, 2070 Route 52, 12533, Hopewell Junction, NY, USA. 


\section{References}

1. Bleck W, Grossterlinden R, Lotter U, Reip CP (1991) Textures in steel sheets. Steel Res 62(12): 580-586. ISSN 0177-4832

2. Bodin A, Sietsma J, van der Zwaag S (2002) Texture and microstructure development during intercritical rolling of low-carbon steels. Metallurg Mater Trans A 33(6): 1589-1603. ISSN 1073-5623

3. Hutchinson B (1999) Deformation microstructures and textures in steels. Philos Trans R Soc Lond A 357(1756): 1471-1485. ISSN 1364-503X

4. Kestens L, Jonas JJ (1999) Deep drawing textures in low carbon steels. Metals And Mater-Korea 5(5): 419-427. ISSN 1225-9438

5. Wronski S, Wrobel M, Baczmanski A, Wierzbanowski K (2013) Effects of cross-rolling on residual stress, texture and plastic anisotropy in f.c.c. and b.c.c. metals. Mater Characterization 77: 116-126. ISSN 1044-5803

6. Yerra SK, Vankudre HV, Date PP, Samajdar I (2004) Effect of strain path and the magnitude of prestrain on the formability of a low carbon steel: On the textural and microtextural developments. J Eng Mater Technol-Trans ASME 126(1): 53-61. ISSN 0094-4289

7. Creuziger A, Hu L, Gnäupel-Herold T, Rollett AD (2013) Data citation: crystallographic texture evolution in 1008 steel sheet during multi-axial tensile strain paths. http://hdl.handle.net/11115/231 Accepted 11 Dec 2013

8. Oberg E, Jones FD, Horton HL, Ryffel H H (1996) Machinery's handbook, 25th edition. Industrial Press Inc., 32 Haviland Street, Unit 2C | South Norwalk, CT 06854

9. (2013) MatWeb. AISI 1008 steel, CQ, DQ, and DQSK sheet. http://www.matweb.com/search/datasheet.aspx? MatGUID=145867c159894de286d4803a0fc0feOf

10. Ray RK, Jonas JJ (1990) Transformation textures in steels. Int Mater Rev 35(1): 1-36. ISSN 0950-6608

11. Kestens L, Jonas JJ (2006) ASM handbook, volume 14A:685-700. ASM, ASM International Materials Park, OH: 44073-0002

12. Inagaki H (1994) Fundamental aspect of texture formation in low-carbon steel. ISIJ Int 34: 313-321

13. Hutchinson B (1994) Practical aspects of texture control in low carbon steels. Mater Sci Forum 157-162: 1917-1928

14. Inagaki H, Kurihara K, Kozasu I (1977) Influence of crystallographic texture on strength and toughness of control-rolled high-tensile strength steel. Trans Iron And Steel Institute of Japan 17(2): 75-81. ISSN 0021-1583

15. Luzin V, Banovic S, Gnaupel-Herold T, Prask HJ, Ricker RE (2005) Measurement and calculation of elastic properties in low carbon steel sheet. In: Houtte PV, Kestens L (eds) ICOTOM 14: Textures Of Materials, volume 495-497 of Materials Science Forum, pp 1591-1596

16. Mirshams RA, Crosby KE, Mohamadian HP, Burris CL (1993) The influence of biaxial stretching on the texture of ultra-low carbon (ulc) steel sheets. Scripta Metallurgica Et Materialia 29(4): 433-438. ISSN 0956-716X

17. Baczynski J, Jonas JJ (1996) Texture development during the torsion testing of alpha-iron and two IF steels. Acta Mater 44(11): 4273-4288. ISSN 1359-6454

18. Kang JY, Bacroix B, Regle H, Oh KH, Lee HC (2007) Effect of deformation mode and grain orientation on misorientation development in a body-centered cubic steel. Acta Mater 55 15: 4935-4946. ISSN 1359-6454

19. Savoie J, Jonas JJ (1994) Simulation of the deformation textures induced by deep-drawing in extra low-carbon steel sheets. Acta Metallurgica Et Materialia 42(12): 4101-4116. ISSN 0956-7151

20. Toth LS, Jonas JJ, Daniel D, Ray RK (1990) Development of ferrite rolling textures in low-carbon and extra low-carbon steels. Metallurgical Trans A 21(11): 2985-3000. ISSN 0360-2133

21. Raabe D (1995) Simulation of rolling textures of b.c.c. matals considering grain interactions and crystalline slip on $\{100\},\{112\}$ and $\{123\}$ planes. Mater Sci Eng A 197: 31-37

22. ASTM International (2008a) Standard test method for atomic emission vacuum spectrometric analysis of carbon and low-alloy steel. ASTM Standards E 415

23. ASTM International (2011) Standard test methods for determination of carbon, sulfur, nitrogen, and oxygen in steel, iron, nickel, and cobalt alloys by various combustion and fusion techniques. ASTM Standards E 1019

24. ASTM International (2004) Standard test methods for determining average grain size E 112. ASTM Standards E 112

25. ASTM International (2008b) Standard test methods for tension testing of metallic materials E8/ E8M. ASTM Standards E 8

26. Foecke T, ladicola MA, Lin A, Banovic SW (2007) A method for direct measurement of multiaxial stress-strain curves in sheet metal. Metallurgical and Materials Transactions A 38A: 306-313

27. Raghavan KS (1995) A simple technique to generate in-plane forming limit curves and selected applications. Metallurgical and Mater Trans A 26A: 2075-2084

28. Brand PC, Prask HJ, Gneaupel-Herold T (1997) Residual stress measurements at the NIST reactor. Physica B 241-243: 1244-1245

29. Matthies S, Wenk HR (1992) Optimization of texture measurements by pole figure coverage with hexagonal grids. Physica Status Solidi (a) 133: 253-257

30. Gnaeupel-Herold T (2012) Bt8 data analysis. http://www.ncnr.nist.gov/instruments/bt8/BT8DataAnalysis.htm. Accessed 29 Jan 2013

31. Kallend UF, John S, Kocks, Rollett AD, Wenk HR (1991) popLA - an integrated software system for texture analysis. Textures and Microstructures 14: 1203-1208

32. Matthies S, Vinel GW (1982) On the reproduction of the orientation distribution function of texturized samples from reduced pole figures using the conception of a conditional ghost correction. Physica Status Solidi B 112(2): K111-K114. ISSN 0370-1972

33. Hielscher R, Schaeben H (2008) A novel pole figure inversion method: specification of the MTEX algorithm. J Appl Crystallography 41: 1024-103

34. Creuziger A, Syed K, Gnaeupel-Herold T (2014) Measurement of uncertainty in orientation distribution function (odf) calculations. Scripta Mat 72-73: 55-58

35. Bunge HJ, Haessner F (1968) 3-dimensional orientation distribution function of crystals in cold-rolled copper. J Appl Phys 39(12): 5503-5515

36. Lebensohn RA, Tomé CN (1993) A self-consistent anisotropic approach for the simulation of plastic deformation and texture development of polycrystals: application to zirconium alloys. Acta Metallurgica et Materialia 41: 2611-2624 
37. Hu L, Rollett AD, ladicola M, Foecke T, Banovic S (2012) Constitutive relations for AA 5754 based on crystal plasticity. Metallurgical and Mater Trans A 43A: 854-869

38. Dawson PR, MacEwen SR, Wu PD (2003) Advances in sheet metal forming analyses: dealing with mechanical anisotropy from crystallographic texture. Int Mater Rev 48: 86-122

39. Pokharel R, Lind J, Kanjarala AK, Lebensohn RA, Li SF, Kenesei P, Suter RM, Rollett AD (2013) Polycrystal plasticity: comparison between grain scale observations of deformation and simulations. submitted to Annual Reviews Condensed Matter Physics

40. Li SF, Lind J, Hefferan CM, Pokharel R, Lienert U, Rollett AD, Suter RM (2012) Three-dimensional plastic response in polycrystalline copper via near-field high-energy X-ray diffraction microscopy. J Appl Crystallography 45: 1098-1108

doi:10.1186/2193-9772-3-1

Cite this article as: Creuziger et al:: Crystallographic texture evolution in 1008 steel sheet during multi-axial tensile strain paths. Integrating Materials and Manufacturing Innovation 2014 3:1.

\section{Submit your manuscript to a SpringerOpen ${ }^{\circ}$} journal and benefit from:

- Convenient online submission

- Rigorous peer review

- Immediate publication on acceptance

- Open access: articles freely available online

- High visibility within the field

- Retaining the copyright to your article

Submit your next manuscript at $>$ springeropen.com 